\title{
Numerical solution of mean field problem with limited management resource
}

V. S. Kornienko, V. V. Shaydurov

Institute of Computational Modeling SB RAS

Email: vika-svetlakova@yandex.ru

DOI 10.24412/cl-35065-2021-1-00-30

Here we propose the computational algorithm for the formulation of MFG problem with the limited management resource during control realization. The Mean Field equilibrium leads to a coupled system of two parabolic partial differential equations: Fokker-Planck-Kolmogorov and Hamilton-Jacobi-Bellman ones with an additional constraint in the form of the inequality. Based on the Karush-Kuhn-Tucker theorem [1], this statement is reduced to finding the saddle point of the Lagrangian with an additional condition of complementary slackness. To approximate the problem, the (Euler-Lagrange) difference schemes are used, which is presented in [2]. An iterative algorithm is presented for solving the obtained discrete problem with justification of the convergence of its elements. The convergence of the algorithm as a whole is illustrated by a numerical example.

This work was supported by the Russian Science Foundation under grant № 20-61-46017.

References

1. Kuhn H.W., Tucker A.W. Nonlinear programming // Second Berkeley Symposium Math. Statistics and Probability, Univ. of California, Berkeley. 1951. P. 481-492.

2. Shaydurov V., Kornienko V., Zhang S. The Euler-Lagrange Approximation of the Mean Field Game for the Planning Problem // Lobachevskii J. Math. 2020. V. 41. P. 2703-2714.

\section{On the accuracy of semi-discrete central-upwind schemes}

O. A. Kovyrkina ${ }^{1,2}$, A. Kurganov ${ }^{3}$, V. V. Ostapenko ${ }^{1,2}$

${ }^{1}$ Lavrentyev Institute of Hydrodynamics SB RAS

${ }^{2}$ Novosibirsk State University

${ }^{3}$ Southern University of Science and Technology, Shenzhen, China

Email: olyana@ngs.ru

DOI 10.24412/cl-35065-2021-1-02-87

On the basis of the method proposed in [1], we studied the accuracy of the second-order semi-discrete finite-volume central-upwind scheme [2] in calculations of shocks propagating with variable velocity. It is shown that in the shock influence domain, the scheme orders of the integral and local convergence decrease to the first one, while on the smooth solutions this scheme has the second order of accuracy. Test calculations are presented.

The reported study was funded by RFBR and NSFC, project number 21-51-53012 (RFBR) and 1201101343 (NSFC).

References

1. Kovyrkina O.A., Ostapenko V.V. On the convergence of shock capturing difference schemes // Dokl. Math. 2010. V. 82 (1). P. 599-603.

2. Kurganov A., Lin C.-T. On the reduction of numerical dissipation in central-upwind schemes // Commun. Comput. Phys. 2007. V. 2 (1). P. 141-163. 